\title{
Retraction Note to "SDF-1/CXCR4 Axis Regulates Cell Cycle Progression and Epithelial-Mesenchymal Transition via Up-regulation of Survivin in Glioblastoma"
}

\author{
Anyan Liao ${ }^{1}$ - Ranran $\mathrm{Shi}^{2} \cdot$ Yuliang Jiang ${ }^{1} \cdot$ Suqing Tian ${ }^{1} \cdot$ Panpan $\mathrm{Li}^{3} \cdot$ Fuxi Song ${ }^{3}$.

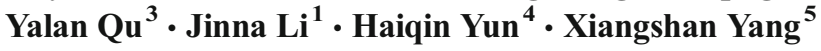

Published online: 18 April 2017

(C) Springer Science+Business Media New York 2017

\section{Retraction Note to: Mol Neurobiol (2016) 53:210-215 DOI 10.1007/s12035-014-9006-0}

This article has been retracted at the request of the Editor-in-Chief and the Publisher per the Committee on Publication Ethics guidelines. The article shows evidence of irregularities in authorship during the submission process; there is strong reason to believe that the peer review process was compromised and the article shows similarities with the following articles which were submitted within a close timeframe:

Wenliang Chen, Xiao Zhong, Yi Wei, Yun Liu, Quan Yi, Genshui Zhang, Lishan He, Fajiang Chen, Yingping Liu, Jiandong Luo, TGF- $\beta$ Regulates Survivin to Affect Cell Cycle and the Expression of EGFR and MMP9 in

The online version of the original article can be found at http://dx.doi.org/ 10.1007/s12035-014-9006-0.

Anyan Liao

liaoanyan323@126.com

1 Department of Radiation Oncology, Peking University Third Hospital, No. 49 Hua Yuan Bei Lu, Beijing 100191, China

2 Department of General Surgery, Shandong Provincial Hospital, Shandong University, Jinan, Shandong, China

3 Qilu Hospital, Shandong University School of Medicine, Shandong University, Jinan, Shandong, China

4 Department of Pathology, Qilu Hospital, Shandong University, Jinan, Shandong, China

5 Department of Pathology, Affiliated Hospital of Shandong Academy of Medical Sciences, Jinan, Shandong, China
Glioblastoma, Molecular Neurobiology, April 2016, Volume 53, Issue 3, pp 1648-1653, DOI: 10.1007/ s12035-015-9121-6; Received: 2 December 2014

Peng Yang, Gang Wang, Hongjun Huo, Qiang Li, Yan Zhao, Yuanhang Liu, SDF-1/CXCR4 signaling upregulates survivin to regulate human sacral chondrosarcoma cell cycle and epithelial-mesenchymal transition via ERK and PI3K/AKT pathway, Med Oncol (2015) 32:377 DOI: 10.1007/s12032-014-0377-x; Received: 13 November 2014

The article "SDF-1/CXCR4 Axis Regulates Cell Cycle Progression and Epithelial-Mesenchymal Transition via Upregulation of Survivin in Glioblastoma" was received 1 November 2014. 